\title{
Analysis of Signal Transmission Characteristics on Meshed Ground Plane
}

\author{
Yun-Chan Nam and Moonjung Kim \\ Department of Electrical Electronic Control Engineering, Kongju National \\ University, Republic of Korea \\ mjkim@kongju.ac.kr
}

\begin{abstract}
In this paper, the signal integrity and power integrity characteristics of a mesh pattern on the ground plane are investigated. The meshed planes are formed with a repetitive width and gap in a printed circuit board (PCB). The size of the square mesh is determined by considering the operating speed and the wavelength of the signal. The spacing between the mesh patterns is kept constant at $0.1 \mathrm{~mm}$, while the width is set to $0.15 \mathrm{~mm}, 0.4 \mathrm{~mm}$ and $0.8 \mathrm{~mm}$, respectively. The calculations such as $S$-parameter, inductance/capacitance extraction, characteristic impedance, eye-diagram and Time Domain Reflectometry $(T D R)$ were performed for analyzing the effects of the meshed ground plane on the signal transmission characteristic in the PCB. S-parameters including the insertion loss and the reflection loss show the different results as the mesh size changes. From the results of characteristic impedance and TDR analysis, the characteristic impedance of the signal line increases with the mesh size and then signal transmission characteristic of the signal line was improved as its impedance is closer to $50 \Omega$. Impedance analysis between powerground planes was carried out. Even though the area of the ground plane is significantly reduced by the mesh pattern, the variation of the power-ground capacitance is less than $8.5 \%$. The PCBs with a low characteristic impedance and a large line width are expected to make closer to impedance matching using the meshed ground and then improve their signal quality and maintain the power integrity.
\end{abstract}

Keywords: Printed Circuit Board, Mesh Pattern, Signal Integrity, Power Integrity

\section{Introduction}

A printed circuit board (PCB) is a substrate for mounting electronic circuit components and serves to connect the components to each other. Like the research trend of electronic devices, PCBs have also been developed with increased degree of integration and more interlayer structures. As electronic devices and systems are becoming smaller, faster and more integrated, signal integrity and power integrity in a PCB are attracting much more attention due to reflection loss, crosstalk and ground bounce. In order to solve these problems, the researches such as impedance matching and simultaneous switching noise (SSN) reduction have been reported [1-2]. In this paper, the analysis for signal and power qualities is performed on a PCB.

One of the important factors affecting the stability of signal transmission and power supply in PCBs are ground plane. It is generally designed in a wide plane configuration. However, in this paper, the mesh structure is introduced in the design of the ground plane and we analyze its effects on the ground plane. It is known that the ground structure with the mesh shape has the advantage of improving the reliability of the PCB and strengthening the external impact by improving the adhesion between the conductors and the dielectric layers.

A number of studies have been conducted on the electromagnetic properties of the meshed ground. It has been reported that applying the mesh structure can suppress 
crosstalk between the microstrip lines and improve signal propagation characteristics at multi-chip package (MCP) [3-4]. In applications of wireless communications, research on resonance and radiation patterns for the meshed ground plane has been carried out. Several studies have been published that it increases near-field coupling and far-field emission [5-9]. However, in this paper, we analyze the effect of signal integrity and power integrity on PCB as the mesh size changes on the ground plane.

\section{Structure and Design of Mesh}

\section{Structure of Mesh Pattern}

Analysis of signal integrity and power integrity was performed on the PCB for semiconductor package. The size of the PCB is $12 \mathrm{~mm} \times 6 \mathrm{~mm}$ and it has a four-layer structure. Signal lines are placed on top and bottom layers. They are connected through vias between layers. The power plane and the ground plane are arranged on the inner layer of the PCB. All conductors are made of copper and the dielectric layers are made of FR4 epoxy-based material. Table 1 shows the layer structure of the PCB and its thickness.

Table 1. Layer Structure of Printed Circuit Board

\begin{tabular}{|l|c|l|}
\hline \multicolumn{1}{|c|}{ Layer } & Thickness $(\mathbf{m m})$ & \multicolumn{1}{c|}{ Material } \\
\hline Top layer & 0.018 & Copper \\
\hline Dielectric & 0.04 & FR4 epoxy \\
\hline Second layer & 0.018 & Copper \\
\hline Dielectric & 0.06 & FR4 epoxy \\
\hline Third layer & 0.018 & Copper \\
\hline Dielectric & 0.04 & FR4 epoxy \\
\hline Bottom layer & 0.018 & Copper \\
\hline
\end{tabular}

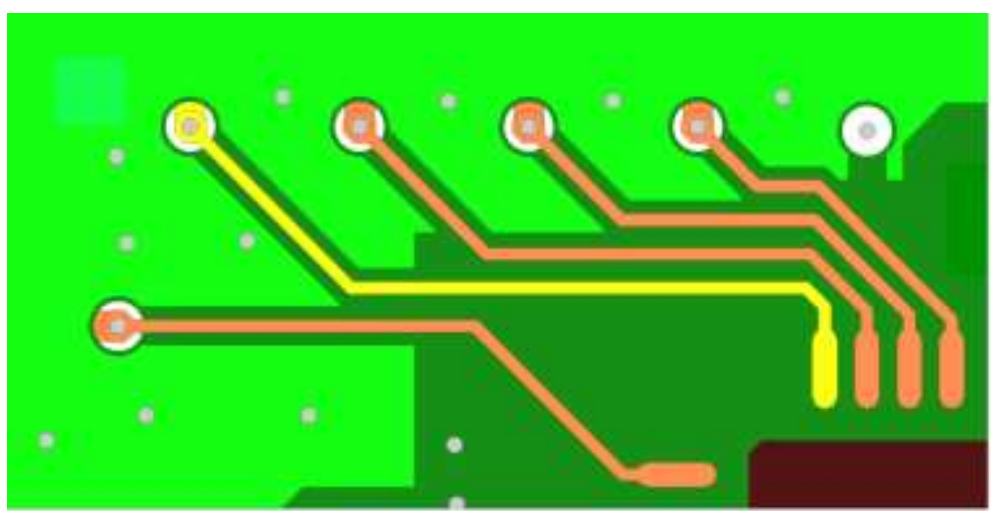

Figure 1. Routing of Printed Circuit Board

As shown in Table 1, the PCB has four layers and has a symmetrical structure with respect to the center dielectric layer. The thickness of all conductors including the signal line, the ground plane and the power plane is $18 \mu \mathrm{m}$. The dielectric layer has a thickness of $40 \mu \mathrm{m}$ or $60 \mu \mathrm{m}$. Figure 1 shows the signal wiring on the PCB. All signal lines are connected through the vias. The ground planes on the top layer and the second layer are also connected by vias. 


\section{Length and Width of Signal Line}

The signal lines are connected from the chip pad of the top layer to the pad of the bottom layer. In addition, all traces are designed as a single-ended line with a width of 0.1 $\mathrm{mm}$. The ground plane on second layer is designated as a reference plane. The length of the signal line is about $10 \mathrm{~mm}$ when that of the pad and the via are included. Figure 2 shows one of the signal lines used in the simulation and the other lines and planes are not shown.

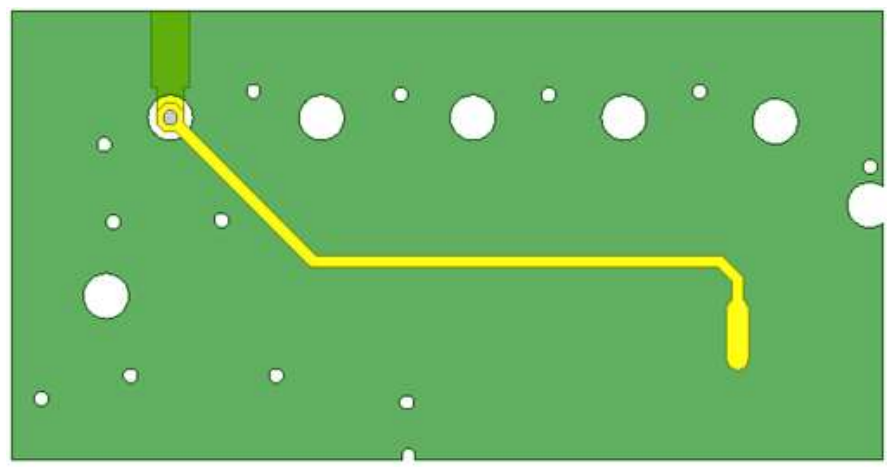

(a) Top view

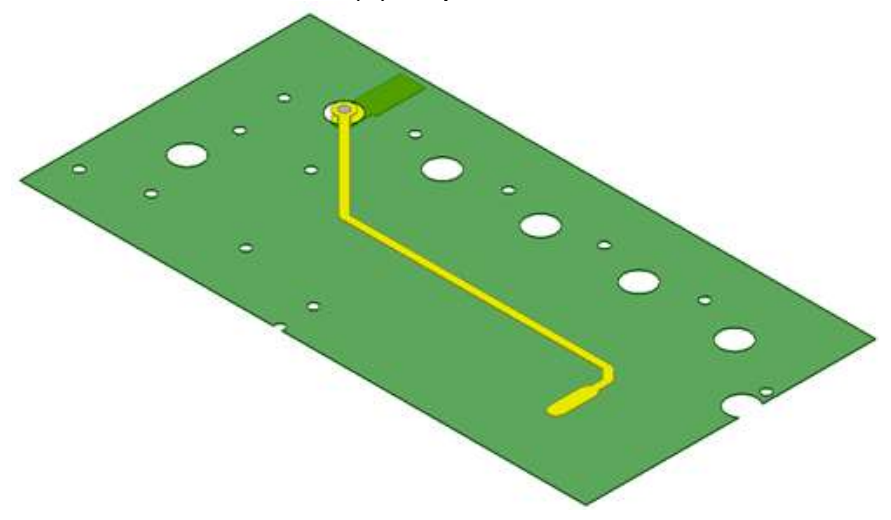

(b) Side view

Figure 2. Top View and Side View of Signal Line on Solid Ground Plane

\section{Design of Meshed Ground Plane}

When the size of the mesh on the ground plane is equal to or larger than the wavelength of the signal, an electromagnetic wave can be radiated from the outside of the ground plane. Therefore, the mesh size should be designed to be sufficiently smaller than the wavelength [10]. When the operating frequency of the signal on the PCB is $200 \mathrm{MHz}$, its wavelength is calculated from Equation 1.

$$
\lambda=\frac{\mathrm{c}}{\mathrm{n} \times \mathrm{f}}=\frac{\mathrm{c}}{\sqrt{\varepsilon_{\mathrm{r}}} \times \mathrm{f}}
$$

where $\lambda$ is the wavelength, $f$ is the operating frequency, $c$ is the speed of light in a vacuum, $\mathrm{n}$ is the refractive index in the dielectric layer and $\varepsilon_{\mathrm{r}}$ is the dielectric constant. The dielectric material of the PCB has a dielectric constant of 4.4. When the signal is operating at the frequency of $200 \mathrm{MHz}$, its wavelength is calculated to be about $0.7 \mathrm{~m}$. The wavelength can be calculated by the effective bandwidth of the digital signal, which is the fifth harmonic of the operating frequency, in other words, $1 \mathrm{GHz}$. Also, considering 
the design limitation that the mesh size should be small enough, the mesh size is determined by applying the $\lambda / 10$ criterion in this paper. Therefore, the minimum mesh size is calculated to be $0.014 \mathrm{~m}$. A square mesh is designed on the ground plane of the PCB. For analyzing the effect of the signal quality on the variation of the mesh size, its length on one side is determined to be $0.15 \mathrm{~mm}, 0.4 \mathrm{~mm}$ and $0.8 \mathrm{~mm}$, respectively. Figure 3 shows the types of the meshed ground plane in this work. The location of the mesh is applied only to the closest ground plane where the signal line passes in.
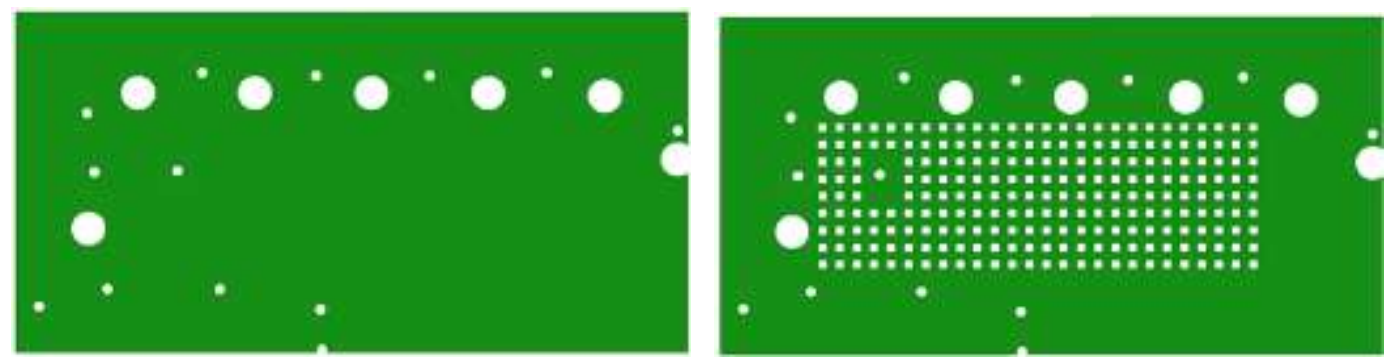

(a) Solid Plane

(b) Meshed Plane with a Square Size of $0.15 \mathrm{~mm}$
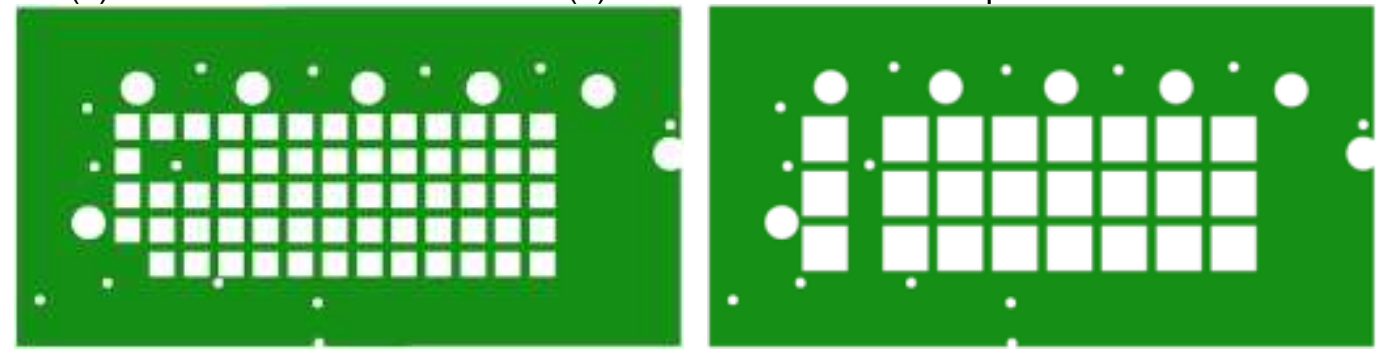

(c) Meshed Plane with a Square Size of $0.4 \mathrm{~mm}$

$0.8 \mathrm{~mm}$

(d) Meshed Plane with a Square Size of

Figure 3. Type of Meshed Ground Plane with Each Mesh Size

\section{Analysis and Result}

Calculations for S-parameter, time domain reflectometry (TDR), characteristic impedance and power-ground impedance are performed for the meshed ground planes as shown in Figure 3. Several simulators such as HFSS, Q3D and Designer are used for the calculations of signal integrity and power integrity. S-parameter analysis, Z-parameter calculation and TDR simulation are carried out using HFSS. In addition, characteristic impedance calculation and eye-diagram analysis are performed using Q3D and Designer, respectively. The frequency range for all analysis is determined based on the fifth harmonic of the operating frequency and then is set up to $1 \mathrm{GHz}$.

\section{Analysis of Signal Integrity}

S-parameters such as insertion loss and return loss are calculated to investigate the signal transmission characteristics of the signal line on the meshed ground plane. Figure 4 shows the calculation result of the return loss. The highest loss is observed in the solid plane. The meshed plane with a square size of $0.15 \mathrm{~mm}$ shows almost the same graph with the solid plane in the all frequency range. But it has slightly lower loss than the solid plane. On the other hand, the meshed planes with a square size of $0.4 \mathrm{~mm}$ and $0.8 \mathrm{~mm}$ show a significant difference in the reflection loss. These results verify that the mesh structure can improve the reflection loss by about $10 \mathrm{~dB}$ at $1 \mathrm{GHz}$. 


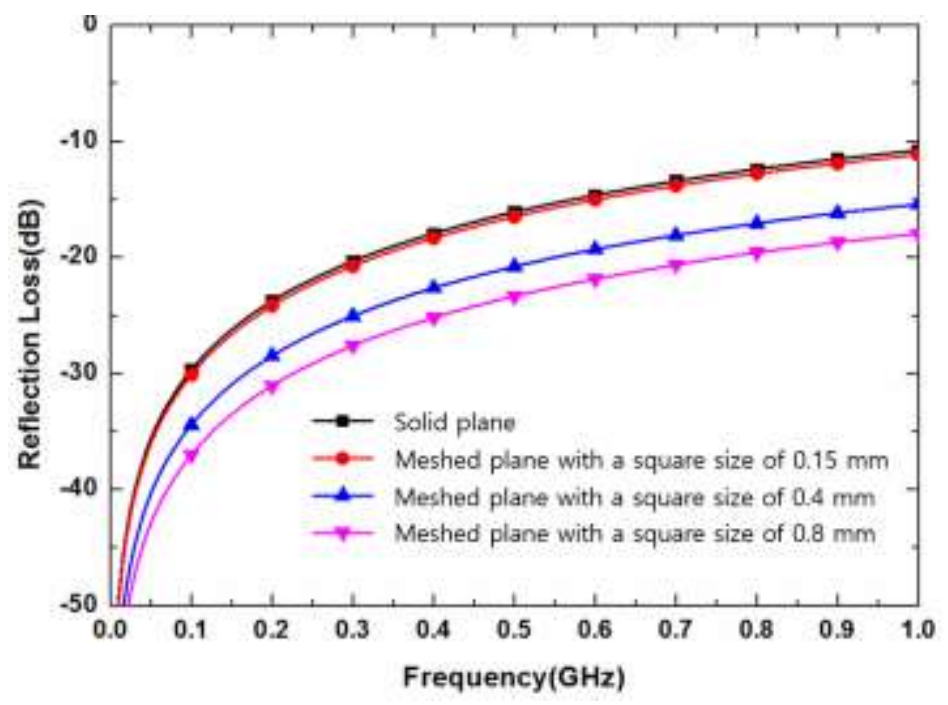

Figure 4. Reflection Loss of Solid and Meshed Planes

The insertion loss for the signal line is shown in Figure 5. It is known that the signal is transmitted and received without almost loss as the insertion loss is closer to $0 \mathrm{~dB}$. As shown in Figure 5, the meshed plane with a square size of $0.8 \mathrm{~mm}$ shows the best performance in the insertion loss. It also shows the three times lower loss result at $1 \mathrm{GHz}$ compared to the solid plane. All meshed planes shows better loss performance than the solid plane in the all frequency range. From these results, it is demonstrated that the insertion loss is further improved as the mesh size on the ground plane increases.

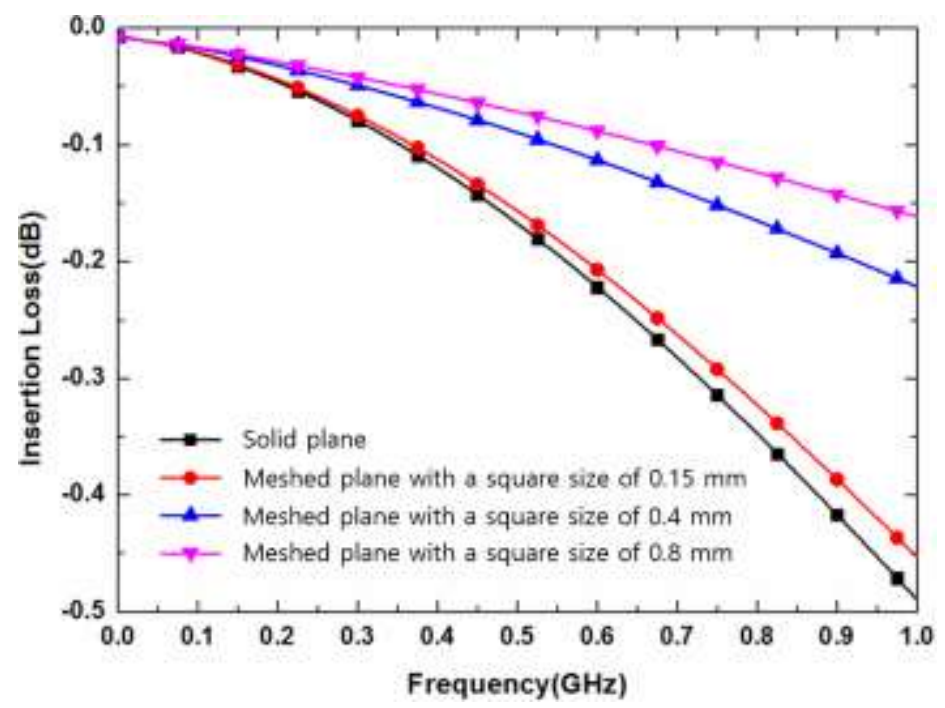

Figure 5. Insertion Loss of Solid and Meshed Planes 


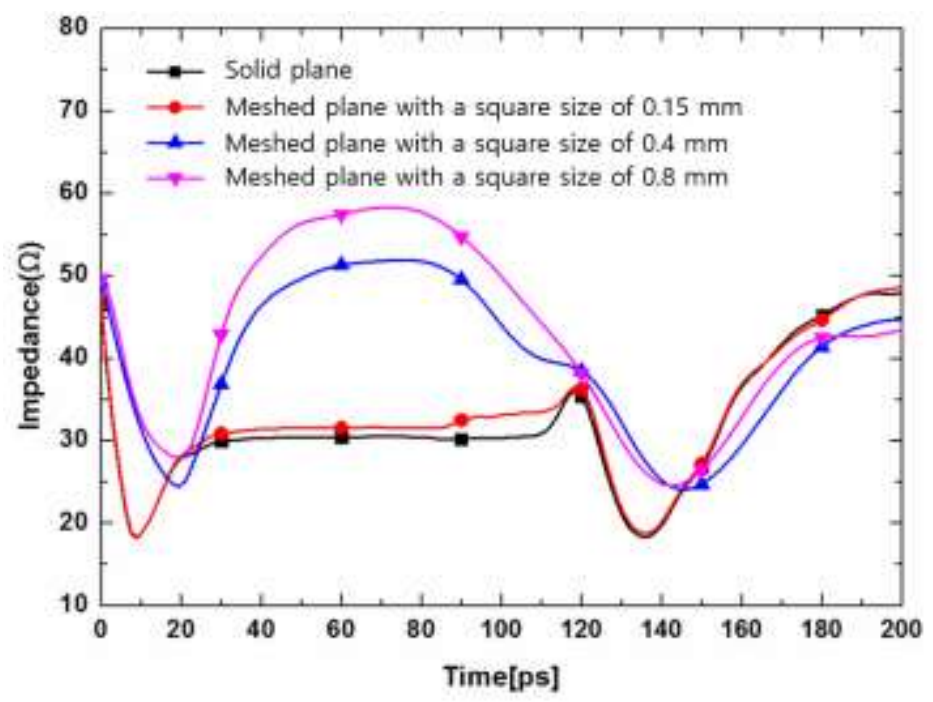

Figure 6. Time Domain Reflectometry of Solid and Meshed Planes

TDR analysis is performed to analyze the characteristic impedance $\left(Z_{0}\right)$ in the time domain. Figure 6 shows the calculation results for TDR simulation. The rise time of the signal line is set to $50 \mathrm{ps}$. The first and second valleys in the TDR graph correspond to the positions of the chip pad and package terminal, respectively. The graphs between both valleys indicate $Z_{0}$ of the trace on the solid and meshed planes. The characteristic impedance of the meshed plane with a square size of $0.15 \mathrm{~mm}$ has approximately $30 \Omega$ and shows slightly higher impedance than that of the solid plane. In case of the mesh size of $0.4 \mathrm{~mm}$ and $0.8 \mathrm{~mm}$, their characteristic impedances are closer to the impedance match. Therefore, their reflection loss and insertion loss are improved by adjusting their characteristic impedances using the mesh structure on the ground plane. It is verified that the signal transmission characteristics can be improved by applying the mesh structure even if the signal line with a low $\mathrm{Z}_{0}$ is used.

Table 2. Capacitance, Inductance and $Z_{0}$ of Solid and Meshed Planes

\begin{tabular}{|l|c|c|c|}
\hline \multicolumn{1}{|c|}{ Plane type } & $\begin{array}{c}\text { Capacitance } \\
(\mathbf{p F})\end{array}$ & $\begin{array}{c}\text { Inductance } \\
(\mathbf{n H})\end{array}$ & $\begin{array}{c}\mathbf{Z}_{\mathbf{0}} \\
(\Omega)\end{array}$ \\
\hline Solid plane without mesh & 2.25 & 2.24 & 31.55 \\
\hline Plane with a mesh size of $0.15 \mathrm{~mm}$ & 2.23 & 2.38 & 32.66 \\
\hline Plane with a mesh size of $0.4 \mathrm{~mm}$ & 1.77 & 3.75 & 46.02 \\
\hline Plane with a mesh size of $0.8 \mathrm{~mm}$ & 1.51 & 4.70 & 55.79 \\
\hline
\end{tabular}

$$
\mathrm{Z}_{0}=\sqrt{\frac{\mathrm{L}}{\mathrm{C}}}
$$

Characteristic impedance analysis is carried out to analyze the reason why the characteristic impedance of the signal line changes according to the mesh size. The capacitance and the inductance of the trace are calculated to use Q3D simulation. Table 2 shows the results of the capacitance and the inductance of the trace as the mesh size changes. The characteristic impedance is calculated from Equation 2. It is known that the capacitance is proportional to the area of the conductor and the inductance is proportional to the return current path on the ground plane. As the mesh size increases, the effective area of the ground plane decreases and then the capacitance also decreases as shown in 
Table 2. The return current path becomes longer as the mesh size increase. Therefore, the inductance also increases as shown in Table 2.

An eye-diagram simulation is performed to investigate the quality of the signal passing through the trace on the solid and meshed ground plane. The rising time and the falling time of the input signal are set to $0.5 \mathrm{~ns}$ and the unit interval is set to $1.2 \mathrm{~ns}$. Figure 7 shows the eye-diagram of the signal line. The eye-diagram is almost the same in both cases of the solid plane and plane with a mesh size of $0.15 \mathrm{~mm}$. However, overshoot and undershoot in the eye-diagram occur at the ground plane with a mesh size of $0.4 \mathrm{~mm}$ and $0.8 \mathrm{~mm}$. From a result of the eye-diagram of the signal line, it is confirmed that the eyediagram is hardly affected in this work even if the mesh size changes. Unlike Sparameters and TDR analysis, there is no significant difference in eye-diagram analysis. The reason is that the signal line's length is a relatively short of about $10 \mathrm{~mm}$ and the mesh size is sufficiently small size based on the operating frequency and the wavelength.
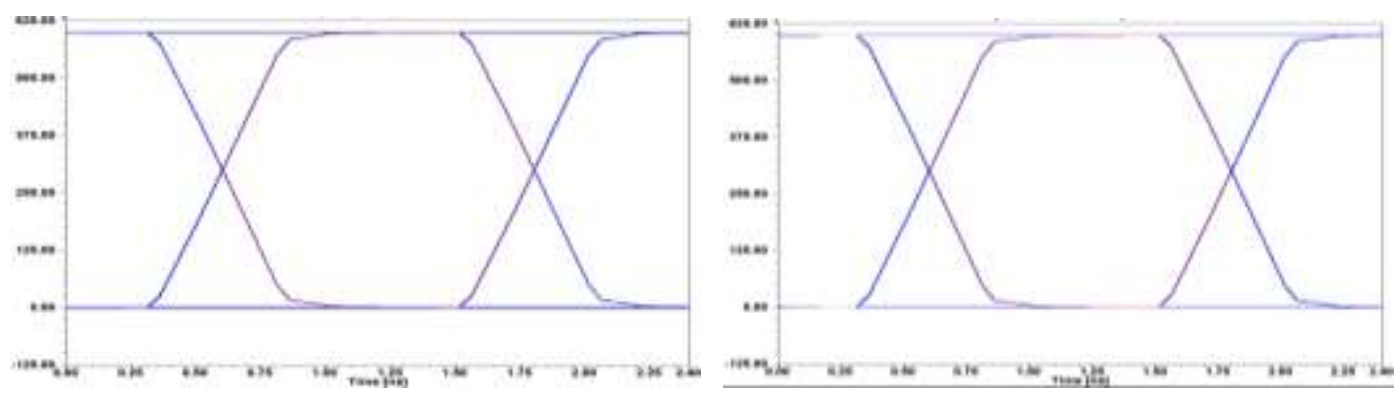

(a) Solid Plane

(b) Meshed Plane with a Square Size of $0.15 \mathrm{~mm}$

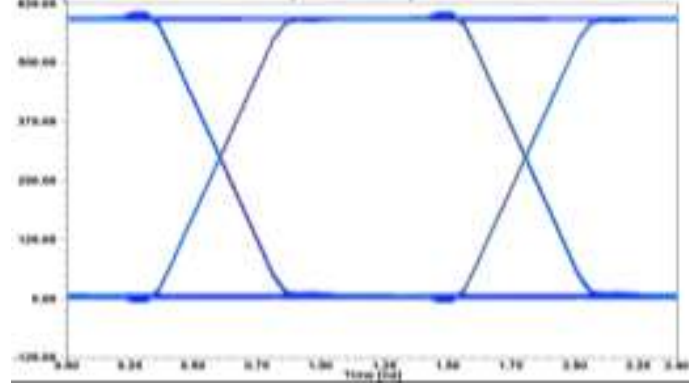

(c) Meshed Plane with a Square Size of $0.4 \mathrm{~mm}$ size of $0.8 \mathrm{~mm}$

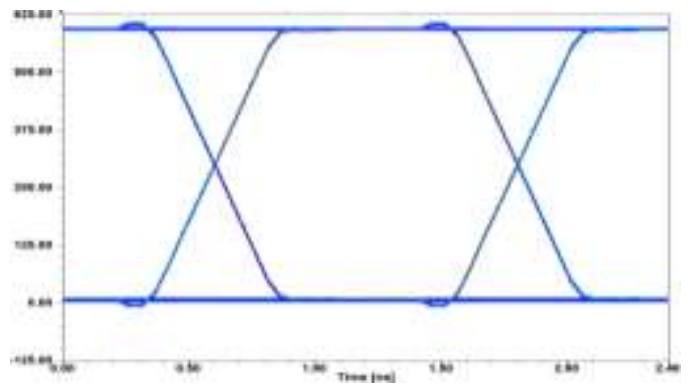

(d) Meshed Plane with a Square

Figure 7. Eye-diagram of Solid and Meshed Planes

\section{Analysis of Power Integrity}

Since the mesh structure is applied to the design of the ground plane, the power-ground impedance changes and then power integrity simulation is performed to analyze this effect. 


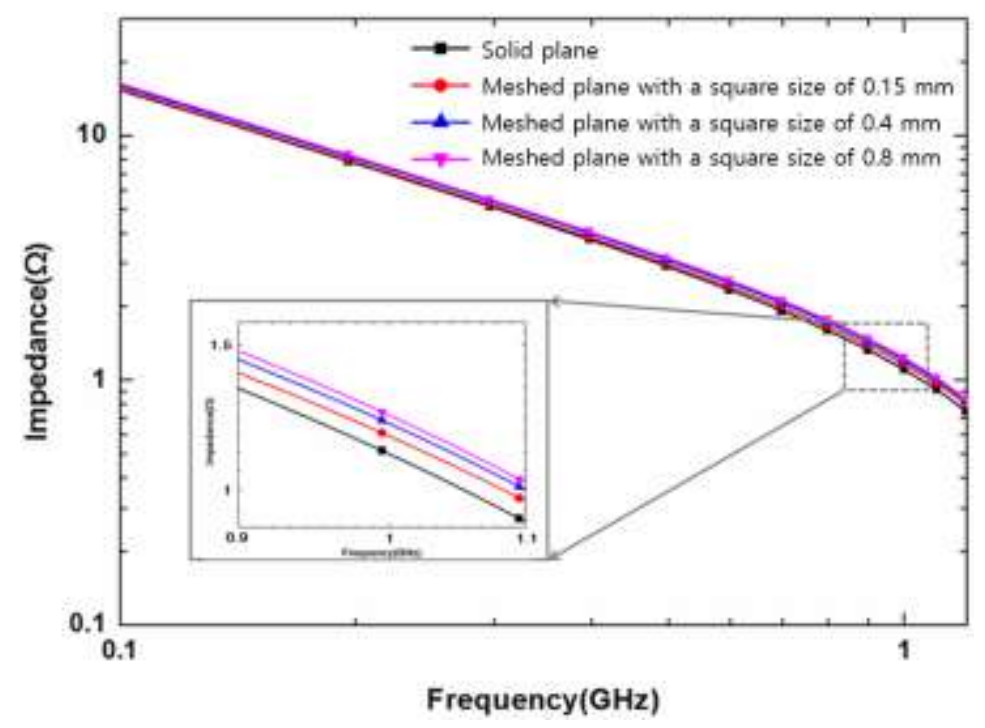

Figure 8. Power-Ground Impedance of Solid and Meshed Planes

The impedance calculation results between the power and ground planes are shown in Figure 8 . As the mesh size increases, the power-ground impedance increases slightly. But, the power-ground impedance has almost the same value despite the large difference in effective area of the ground plane as shown in Figure 3. Therefore, the mesh structure and size in this paper does not substantially affect the power-ground impedance. The change of capacitance between the power and ground plane is analyzed using Q3D simulation as the mesh size increase. Table 3 shows the power-ground capacitance for each mesh size on the ground plane. As the mesh size increases, the area of the ground plane decreases and then the power-ground capacitance decreases in Table 3. However, even though the effective area of the ground plane is significantly reduced by the mesh structure, the variation of the power-ground capacitance is less than $8.5 \%$. But, the mesh pattern can reduce the self-capacitance of the ground and the mutual capacitance between the signal line and the ground plane. Thus it increases the characteristic impedance of the signal line.

\section{Table 3. Power-Ground Capacitance of Each Mesh Size}

\begin{tabular}{|l|c|}
\hline \multicolumn{1}{|c|}{ Plane type } & Capacitance $(\mathbf{p F})$ \\
\hline Solid plane without mesh & 105.46 \\
\hline Plane with a mesh size of $0.15 \mathrm{~mm}$ & 103.92 \\
\hline Plane with a mesh size of $0.4 \mathrm{~mm}$ & 97.66 \\
\hline Plane with a mesh size of $0.8 \mathrm{~mm}$ & 96.46 \\
\hline
\end{tabular}

\section{Conclusion}

In this work, we analyze the effect of signal integrity and power integrity on mesh size in the ground plane of PCB. Mesh pattern is designed to be small enough considering the wavelength and the operating frequency of the signal. The reflection loss and the insertion loss are improved by adjusting the mesh size on the ground plane and then matching the trace's characteristic impedance to $50 \Omega$. Compared to the solid plane, the mesh pattern with a size of $0.8 \mathrm{~mm}$ improves the reflection loss by about $10 \mathrm{~dB}$ at $1 \mathrm{GHz}$ and its insertion loss has the three times lower loss result at $1 \mathrm{GHz}$. However, the eye-diagram and the power-ground impedance are hardly affected in this work even if the mesh size changes. The PCBs with a low characteristic impedance of less than $50 \Omega$ are expected to 
be closer to the impedance match using the mesh structure on the ground plane and then improve their signal quality and maintain the power integrity.

\section{Acknowledgement}

This work was supported by the research grant of the Kongju National University in 2013.

\section{References}

[1] M. Kim, "Signal Transmission Analysis of Burn-In Socket for Fine-Pitch Ball Grid Array Package", International Journal of Control and Automation, vol. 7, (2016), pp. 447-484.

[2] M. Kim, "Electrical Characterization on Signal Transmission of SATA Cable Assembly", International Journal of Control and Automation, vol. 8, (2014), pp. 173-1804.

[3] C.-P. Chien, A. F. Burnett, J. M. Cech and M. H. Tanielian, "The Signal Transmission Characteristics of Embedded Microstrip Transmission Lines Over a Meshed Ground Plane in Copper/Polyimide Multichip Module", vol. 17, (1994), pp. 578-583.

[4] B. Cabon, N. Corrao and J. Chilo, "Electromagnetic Characterisation and Modeling of Stripline Structures with Slotted and Meshed Ground Planes”, European Microwave Conference, (1991), pp. $1562-1567$.

[5] M. Drissi, M. Elkandoussi and G. Kergonou, "A Full-Wave Characterization of PCB Having Various Ground Plane Shapes", IEEE International Symposium on, (2001), pp. 1213-1216.

[6] K. Leong, A. C. Guyette, B. Elamaran, W. A. Shiroma and T. Itoh, "Coupling Suppression in Microstrip Lines Using a Bi-Periodically Perforated Ground Plane", IEEE microwave and wireless component letter, vol. 12, (2002), pp. 169-171.

[7] E. Marzolf and M. Drissi, "Analysis of Transmission Lines over Slotted Ground Planes and Method of Compensating Resonance Effects", Electronic System integration Technology Conference, (2006), pp. 62-66.

[8] U. Choi, Y.-J. Kim and Y.-S. Kim, "Crosstalk Reduction in Printed Circuit Boards Using IrregularlySpaced Vias in a Guard Trace Over a Slotted Ground Plane", IEEE Circuit Theory and Design, (2009), pp. 794-797.

[9] S. Kwak, Y. Jo, J. Jo and S. Kim, "Power Integrity and Shielding Effectiveness Modeling of Grid Structured Interconnects on PCBs", The Institute of Electronics Engineers of Korea, vol. 12, (2012), pp. 320-330.

[10] R. Ulrich, "Far-infrared Properties of Metallic Mesh and Its Complementary Structure", Infrared Physics, vol. 7, (1967), pp. 37-55.

\section{Authors}
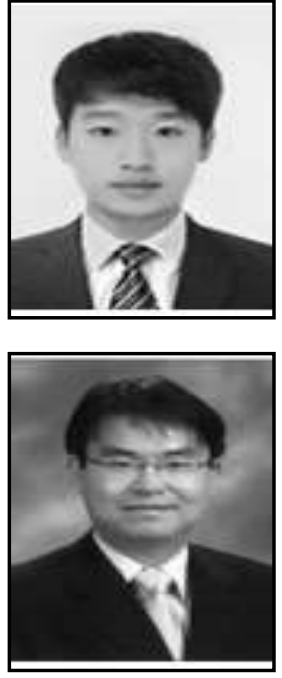

Moonjung Kim, he received the B.S. degrees in electronic engineering from Kyungpook National University, Daegu, Republic of Korea, in 1997 and the M.S. and Ph.D. degree in electrical and electronics engineering from Korea Advanced Institute of Science and Technology, Daejeon, in 2003. Since September 2006, he has been with the Division of Electrical Electronics and Control Engineering, Kongju National University, Chungnam, Korea, as a faculty member. His current research interests include the signal/power integrity and signal transmission analysis in the highspeed package/board/system. 
International Journal of Control and Automation Vol.10, No.8 (2017) 